Corrigendum

\title{
Corrigendum to "Inguinal Herniation of the Urinary Bladder Presenting as Recurrent Urinary Retention"
}

\author{
Amit Frenkel $\mathbb{D}^{1}{ }^{1}$ Aviel Roy-Shapira, ${ }^{1,2}$ Ilan Shelef, ${ }^{3}$ Gadi Shaked, ${ }^{2}$ Evgeni Brotfain $\mathbb{D},{ }^{1}$ \\ Leonid Koyfman, ${ }^{1}$ Abraham Borer, ${ }^{4}$ and Moti Klein ${ }^{1}$ \\ ${ }^{1}$ General Intensive Care Unit, Soroka University Medical Center and the Faculty of Health Sciences, Ben-Gurion University of \\ the Negev, 84101 Beer-Sheva, Israel \\ ${ }^{2}$ Department of General Surgery, Trauma Unit, Soroka University Medical Center and the Faculty of Health Sciences, Ben- \\ Gurion University of the Negev, 84101 Beer-Sheva, Israel \\ ${ }^{3}$ Department of Radiology, Soroka University Medical Center and the Faculty of Health Sciences, Ben-Gurion University of the Negev, \\ 84101 Beer-Sheva, Israel \\ ${ }^{4}$ Infection Control and Hospital Epidemiology Unit, Soroka University Medical Center and the Faculty of Health Sciences, Ben- \\ Gurion University of the Negev, 84101 Beer-Sheva, Israel
}

Correspondence should be addressed to Amit Frenkel; frenkela@prognosa.co.il

Received 3 September 2019; Accepted 16 September 2019; Published 14 October 2019

Copyright (c) 2019 Amit Frenkel et al. This is an open access article distributed under the Creative Commons Attribution License, which permits unrestricted use, distribution, and reproduction in any medium, provided the original work is properly cited.

In the article titled "Inguinal Herniation of the Urinary Bladder Presenting as Recurrent Urinary Retention" [1], there was an error in the Discussion, which should be corrected as follows:

"The great majorities of significant herniations of the bladder occur in elderly men and are likely to be the result of the need to increase contraction force to overcome distal urinary tract obstruction due to an enlarged prostate [4]."

\section{References}

[1] A. Frenkel, A. Roy-Shapira, I. Shelef et al., "Inguinal herniation of the urinary bladder presenting as recurrent urinary retention," Case Reports in Surgery, vol. 2015, Article ID 531021, 3 pages, 2015. 


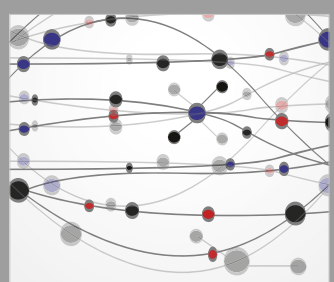

The Scientific World Journal
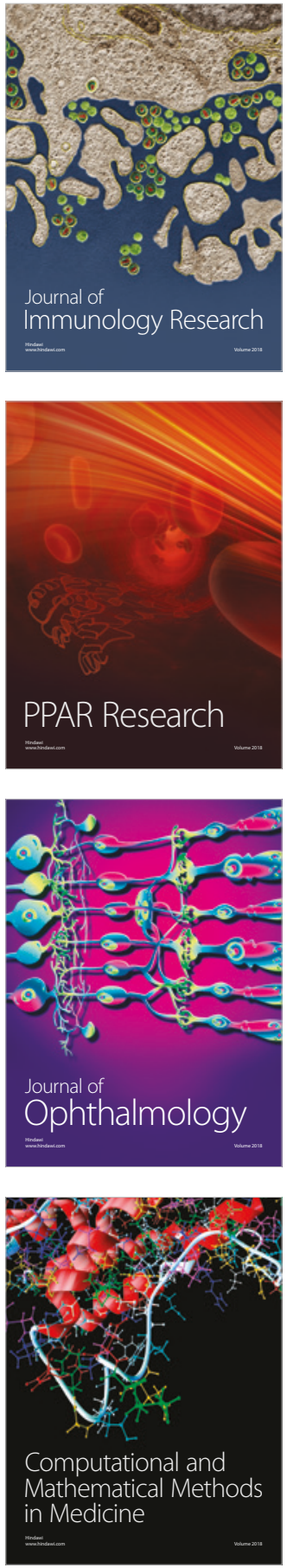

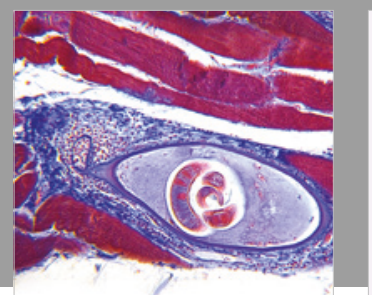

Gastroenterology Research and Practice

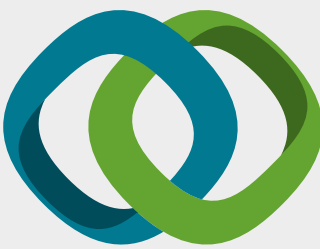

\section{Hindawi}

Submit your manuscripts at

www.hindawi.com
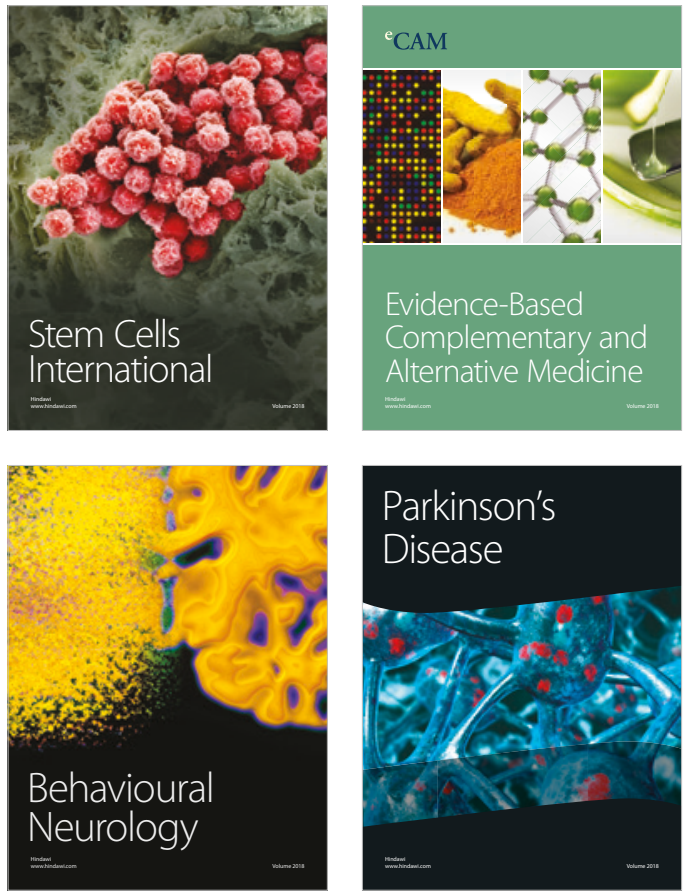

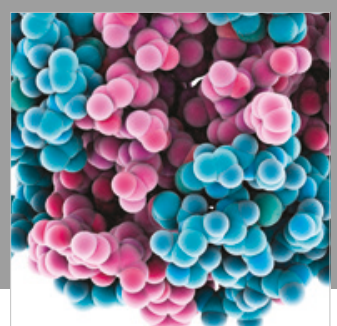

ournal of

Diabetes Research

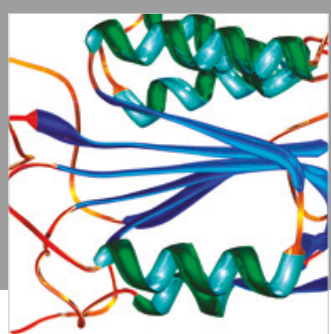

Disease Markers
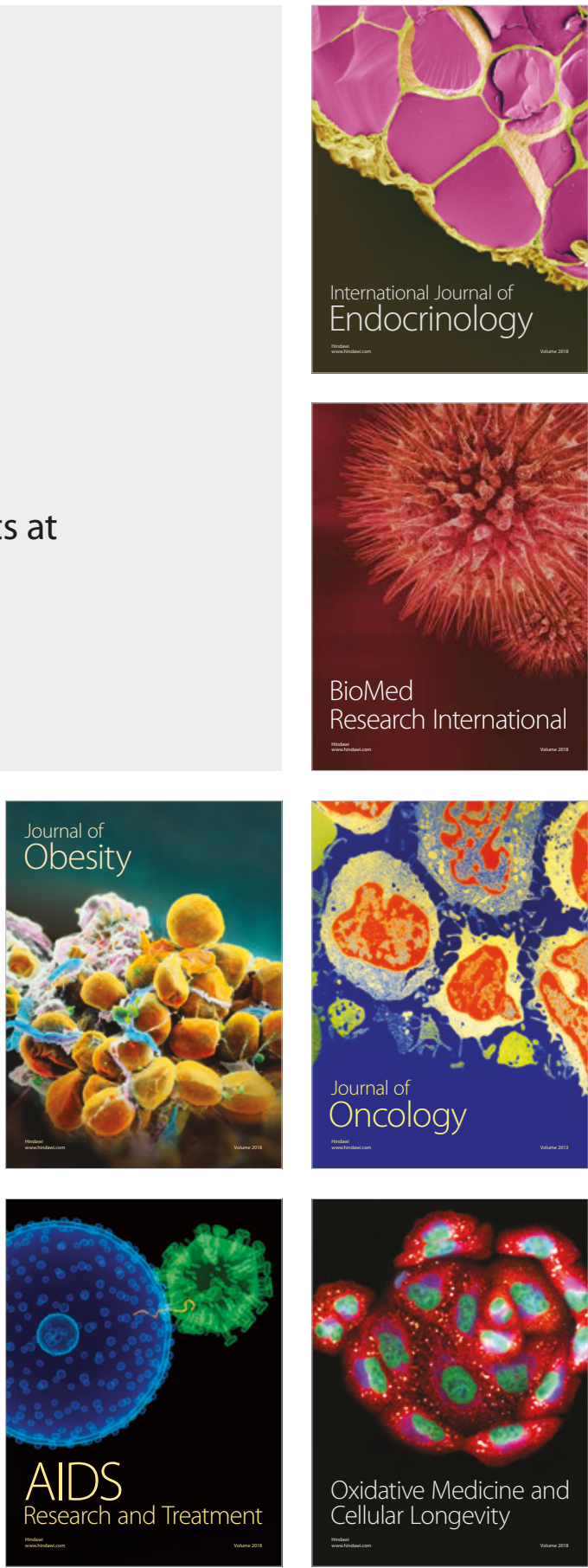